\title{
Malignant adenomyoepithelioma of the breast
}

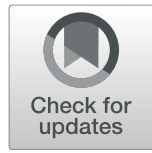

Kazuki Moro ${ }^{* *}$ (D) Eiko Sakata', Asa Nakahara ${ }^{2,3}$, Hideki Hashidate ${ }^{2}$ Emmanuel Gabriel ${ }^{4}$ and Haruhiko Makino ${ }^{1,5}$

\begin{abstract}
Background: Adenomyoepithelioma (AME) of the breast is a very rare tumor and is generally considered to be benign. However, some show malignant transformation, which results in local recurrences or distant metastases. The morphological features of AME that might predict malignant potential have not been elucidated. Moreover, there is also no established multidisciplinary treatment for malignant AME aside from complete excision at an early stage.

Case presentation: A 64-year-old female diagnosed with AME of the left breast underwent lumpectomy. The surgical margins were negative. Six months after the operation, however, malignant AME recurred locally in the left breast. MRI showed multiple masses, which invaded the skin. A left mastectomy with axillary lymph node dissection was performed. Additional areas of AME were found in about one third of the entire breast. Eight months after the mastectomy, lung metastases were detected. She underwent chemotherapy with fluorouracil, epirubicin, and cyclophosphamide (FEC) for 9 cycles with little response. Lung metastasectomy was performed. Nine months after lung metastasectomy, the metastases were widespread to the brain, heart, and kidney; she subsequently died 2 months later.

Conclusions: Malignant AME has various morphological features, and in this report, we characterize new findings from both imaging and pathology/autopsy. Malignant potency is related to the tumor size, tumor appearance, and mitoses, even if only a few. Given that ductal spread is one of the morphological features of malignant AME, it is of paramount importance to assess the surgical margins.
\end{abstract}

Keywords: Differentiation, Ductal spread, Hematogenous spread, Malignant adenomyoepithelioma, Surgical margin

\section{Background}

Adenomyoepithelioma (AME) of the breast is a rare disease characterized by a bicellular pattern consisting of both ductal and myoepithelial cells [1,2]. While most of AMEs of the breast are benign with good prognosis, some have shown malignant transformation [3, 4]. Malignant AME is difficult to differentiate from other benign diseases such as intraductal papilloma, tubular adenoma, and sclerosing adenosis. Moreover, malignant AME has a strong potential for local recurrence and distant metastasis to sites including the lungs [4], thyroid gland [5], bone [6], and brain [3].

Since the morphological features of AME that could predict the malignant potency have not been elucidated, the tumors which seem to be benign have the possibility of changing into malignant tumors. Our case is atypical in that we describe new morphological features not previously reported. Thus, our case of malignant AME is of interest not only for its rarity, but also for the aspects of the morphological features.

\footnotetext{
* Correspondence: kmoro@med.niigata-u.ac.jp

${ }^{1}$ Department of Breast Surgery, Niigata City General Hospital, 463-7

Shumoku, Chuo-ku, Niigata 950-1197, Japan

Full list of author information is available at the end of the article
}

\section{Case presentation}

A 64-year-old female with no significant past medical history was referred to our institution after new microcalcifications were identified in the left breast on 
screening mammography. Diagnostic ultrasonography (US) showed a $4.9 \times 5.1 \times 4.2 \mathrm{~mm}$ low echoic mass on the left between external-inferior and internal-inferior quadrants (Fig. 1a). Only duct papillomatosis was found on core needle biopsy. This was found to be concordant, and she was treated with observation.

Two and a half years after the first consultation, she palpated a mass at the same location. A new US highlighted a larger $26.1 \times 22.6 \times 26.8 \mathrm{~mm}$ low echoic mass (Fig. 1b). Benign adenomyoepithelioma (AME) was identified on core needle biopsy. As the patient was a candidate for breast conservation, lumpectomy was performed. The histological analysis revealed a benign AME with few mitotic figures measuring $31 \times 27 \times 21 \mathrm{~mm}$. All surgical margins were negative (Fig. 1c). The tumor consisted of both epithelial cells positive for CAM 5.2 and myoepithelial cells positive for $\alpha$-smooth muscle actin (SMA) (Fig. 1c).

Six months after the primary operation, she noticed a mass at the same location again. Diagnostic US highlighted a $34 \times 26 \mathrm{~mm}$ hypoechoic mass along the left lumpectomy cavity (Fig. 2a). A computed tomography (CT) scan of the chest, abdomen, and pelvis showed no signs of distant metastasis. MRI showed multiple masses, which invaded the skin. Pectoralis muscle invasion was also suspected (Fig. 3b, c).

We therefore performed a left mastectomy and axillary lymph node dissection. Pathological examination showed a malignant AME with multiple AME tumors collectively measuring $110 \times 105 \times 55 \mathrm{~mm}$ (Fig. 3a). The tumor consisted of both epithelial cells positive for CAM 5.2 and myoepithelial cells positive for SMA. Ki-67 labeling index (Ki-67) was 44\%. The myoepithelial cells were strongly positive for P53 (Fig. 3b). Additional intraductal consistent with AME were spread about one third of the entire breast. Immunohistochemical analysis showed that these lesions were also consistent with AME. Resected lymph nodes $(0 / 26)$ were negative.

Eight months after the secondary operation, chest Xray and CT revealed two nodular masses located in her left upper (measuring $8 \times 7 \mathrm{~mm}$ ) and right upper lobes of the lungs (measuring $10 \times 7 \mathrm{~mm}$ ) (Fig. 4). She underwent chemotherapy with fluorouracil, epirubicin, and cyclophosphamide (FEC) for 9 cycles. Imaging after 9 cycles showed a partial reduction in the lung metastases and no other new distant metastasis. Due to the cardiotoxicity of epirubicin, we elected to perform pulmonary metastasectomies. A left upper lobectomy with video-
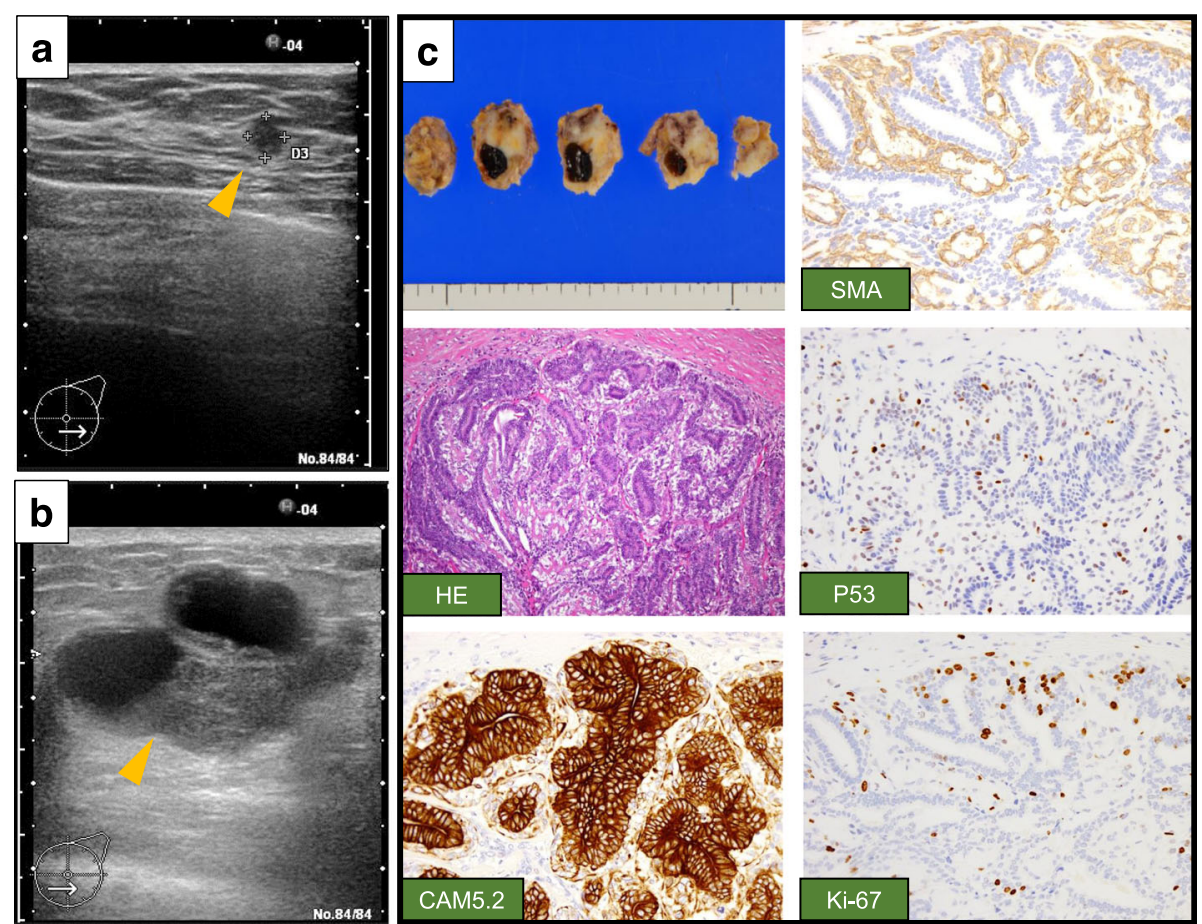

Fig. 1 Ultrasonographic examination from the first consultation to the primary operation. a An ultrasonography (US) scan of the breast demonstrated a low-density area measuring $4.9 \times 5.1 \times 4.2 \mathrm{~mm}$ (orange arrowhead). b An US scan of the breast demonstrated a low-density area measuring $26.1 \times 22.6 \times 26.8 \mathrm{~mm}$ (orange arrowhead). $\mathbf{c}$ Histopathological findings of the tumor at the primary operation. Although mitotic figures were present slightly, there was no proliferative lesion or ductal invasion. The tumor was consisted of both epithelial cells, which was positive for CAM 5.2, and myoepithelial cells, which was strongly positive for a-smooth muscle actin (SMA). Ki-67 labeling index (Ki-67) and P53 were weakly positive. $\times 200$ 

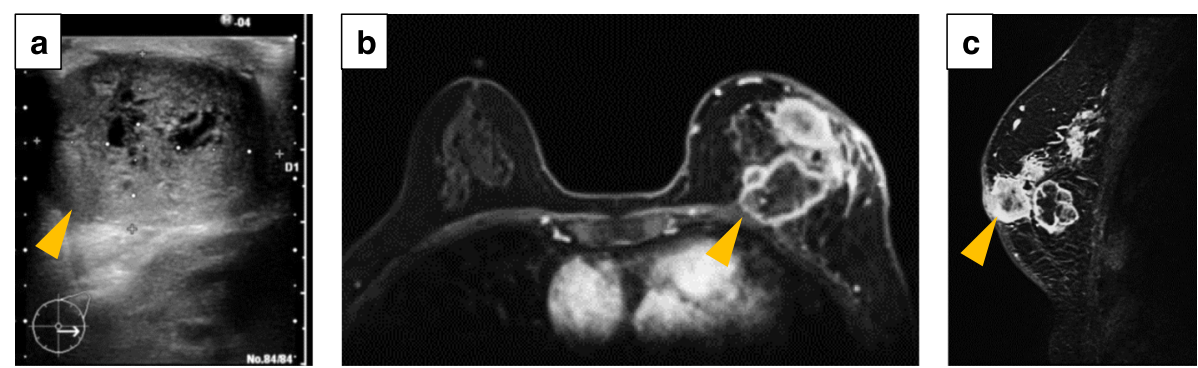

Fig. 2 The data of the secondary operation. a An ultrasonography (US) scan of the breast demonstrated a scale out size of low echoic mass (orange arrowhead). b A magnetic resonance imaging (MRI) showed some tumors including internal necrosis with ductal spread (orange arrowhead). The pectoralis muscle invasion was suspected. c A sagittal sequence of MRI scans showed the wide ductal spread of malignant adenomyoepithelioma (AME), which invaded the skin (orange arrowhead)

assisted thoracic surgery (VATS) and subsequently a right segmentectomy (S2) were performed.

Nine months after the metastasectomy, however, she presented with right lower abdominal pain and dysuria. A whole-body CT scan demonstrated multiple lung metastases, right kidney metastasis, right adrenal metastasis, ovarian metastasis, and abdominal paraaortic node metastasis. She underwent salvage eribulin monotherapy. The whole-body CT after eribulin 3 cycles demonstrated that the diseases continued to progress. Two months later, she had expired. Autopsy demonstrated that the metastasis was widespread to the heart muscle, kidney, and brain (Fig. 5a-c).

\section{Discussion}

The definition of malignant AME is not clearly defined. Nadelman et al. described 2 cases of metastases of histologically "benign" AME of the breast to the lung [7]. Some AME tumors appear benign but may contain cellular atypia or mitotic figures. Although morphological features of malignant transformation include nuclear atypia, increased mitotic activity, necrosis, and infiltrative growth pattern [8], there is no established reference to differentiate between benign and malignant AMEs. In our case, mitotic figures were present in the tumor at the primary operation. Considering the patient's course, this may have provided a clue as to the malignant

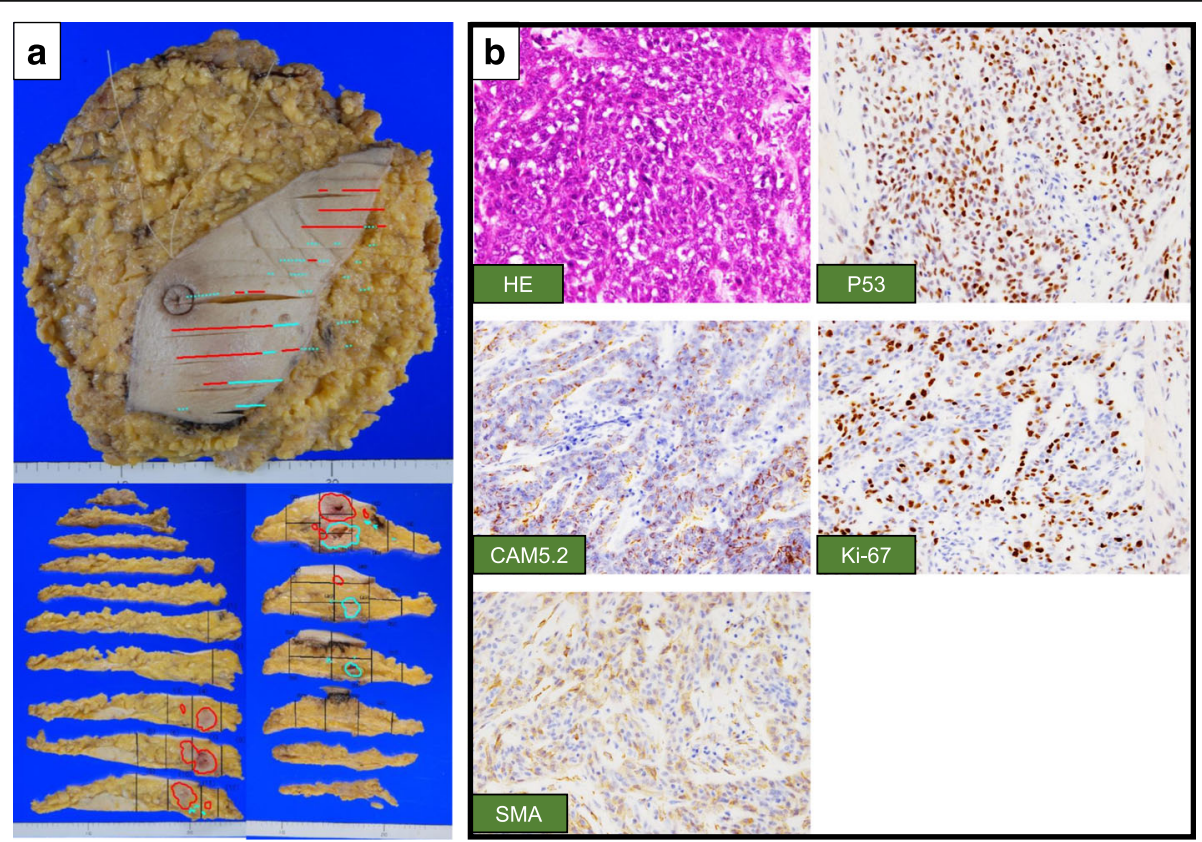

Fig. 3 Histopathology of the malignant adenomyoepithelioma. a Gross specimen of malignant AME. Red markers show malignant lesions, and blue markers show ductal lesions. The size of tumor was $110 \times 105 \times 55 \mathrm{~mm}$. b Histopathology of the malignant AME. The tumor was consisted of both epithelial cells, which was positive for CAM 5.2, and myoepithelial cells, which was positive for a-smooth muscle actin (SMA). Ki-67 labeling index (Ki-67) was 44\%. The myoepithelial cells were strongly positive for P53. × 200 


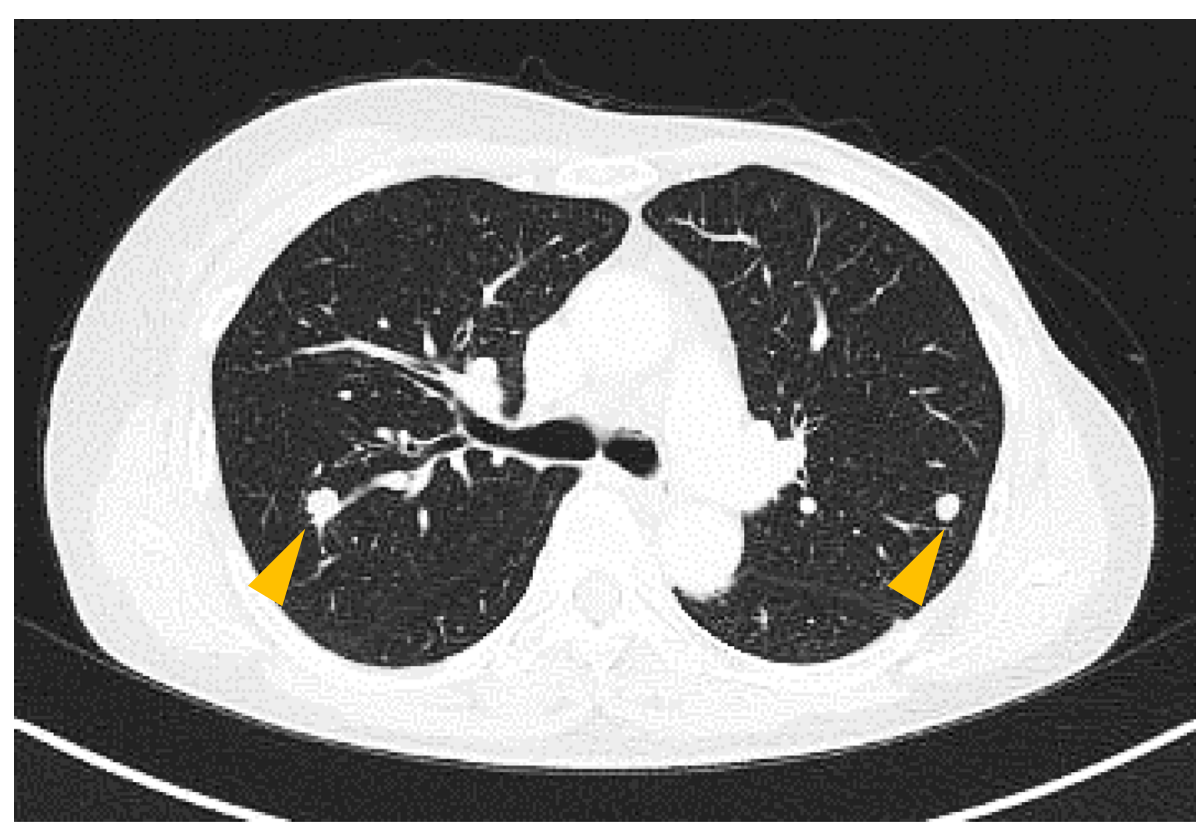

Fig. 4 Images of computed tomography (CT) at diagnosis showing lung metastasis. CT revealed two nodular masses located in the patient's left upper and right upper lobes of the lungs (orange arrowheads)

potential of her primary tumor. Tumor size is also one of the characteristics that may be related to potential malignancy. Patients with a primary tumor of $\geq 16 \mathrm{~mm}$ often presented with metastases [9]. Some papers have concluded that AMEs over $2 \mathrm{~cm}$ should be treated as malignant $[10,11]$. In our case, the size of the tumor at the primary operation was $31 \mathrm{~mm}$, leading to poor prognosis. In addition to the tumor size, tumor appearance is an important factor in prognosis. Generally, malignant AME has been described as a large stable mass [12], but

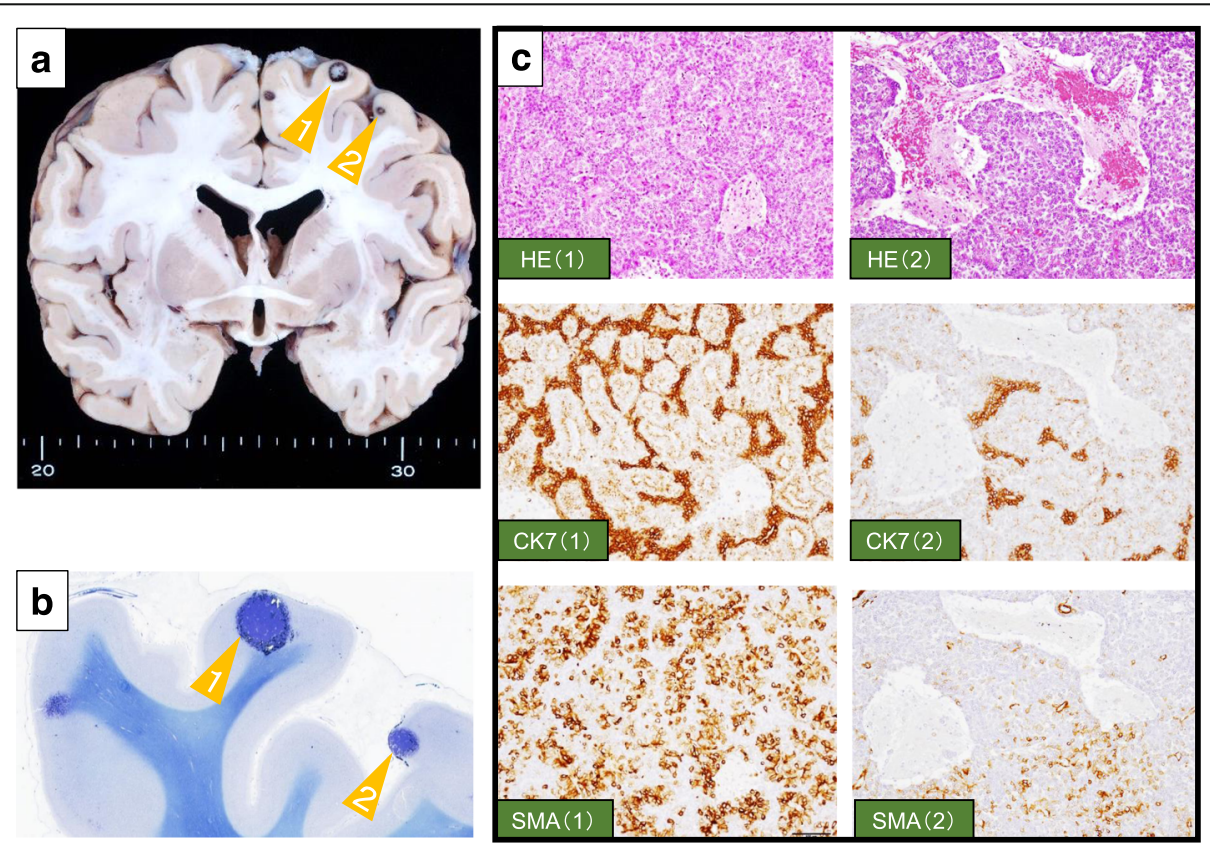

Fig. 5 Images of autopsies. a Gross specimen of brain. There are some metastatic lesions (orange arrowheads). $\mathbf{b}$ Histopathological findings of the brain. The tissue of the brain metastases stained with Klüver-Barrera staining. $\mathbf{c}$ The brain metastases (in a number 1 and number 2) were positive for CK7 and asmooth muscle actin (SMA) retrospectively. The epithelial component was increased in the metastatic lesions compared to the primary breast tumor 
our case of malignant AME showed multifocality within the breast. To the best of our knowledge, this represents an unusual presentation of AME. Other factors including mitotic figures, tumor size, and tumor appearance are also indicators of the malignant potential.

In managing the malignant AME, it is important to recognize their poor prognosis. It is known that P53 and Ki-67 are important prognostic factors. In comparison to immunohistochemical features of malignant AME, cases with higher expressions of P53 and Ki-67 were worse prognostic factors [13]. In our case, both of P53 and Ki-67 were high, leading to the observed poor prognosis. There are also no unified views whether hematogenous spread or lymphatic spread occurs in malignant AME, but metastases to the axillary lymph nodes are extremely rare [12]. The pattern of spread in our case suggests that metastatic malignant AME metastasizes mainly through the hematogenous route rather than lymphatic system.

In general, the biological behavior of tumors developing in mammary glands ranges from benign to malignant transformation of either the epithelial or the myoepithelial component or both. As portrayed by our case, the biological behavior of tumors is different between primary and recurrent lesions. Although all of the lesions consisted of both the epithelial component and the myoepithelial component, the proportions of the two components were different. The epithelial component was most abundant in the primary site (Fig. 1c) followed by the brain metastases (Fig. 5c), and least in secondary site, which was diagnosed as malignant AME (Fig. 3b). Moreover, the proportions of epithelial and myoepithelial cells were different among the brain metastases (Fig. 5c). Recognizing that heterogeneity between the proportions of epithelial and myoepithelial cells impacts treatment resistance [14], the increased proportion of the myoepithelial component compared to the epithelial component likely contributes to worse prognosis.

The treatment of malignant AME is not established except for complete excision at an early stage. Kihara et al. concluded that a complete local excision remains the only way to reduce the chance of local recurrence and distant metastases [4]. On the other hand, it remains unknown whether axillary lymph node sampling is necessary. Similar to surgical treatment, there is no effective adjuvant chemotherapy at present. Chemotherapy has been used in some malignant cases, but the majority of them are not effective $[5,6]$. Lee et al. reported that eribulin had a beneficial effect on malignant AME of the breast with multiple hepatic, pleural, and abdominal wall metastases [15]. Neither complete resection of lung metastases nor chemotherapy including FEC and eribulin could control the malignant AME in our case.
Malignant AME can progress very aggressively as it did in the current case. Even if AME presents in a benign manner, it is important to assess the extent of the primary lesion by MRI and to consider wide surgical margins at the primary operation to perform complete resection as this may be the only potential option for a favorable outcome.

\section{Conclusions}

Malignant AME has various morphological features, and we demonstrated unique findings from both imaging and pathology/autopsy. Even only a few mitotic figures should raise caution regarding the malignant potential of the tumor in addition to the size and appearance of AME. Considering that ductal spread is one of the more aggressive morphological features of malignant AME, it is of paramount importance to assess the surgical margin before resection and obtain widely negative surgical margins.

\section{Abbreviations}

AME: Adenomyoepithelioma; CT: Computed tomography; FEC: Fluorouracil, epirubicin, and cyclophosphamide; Ki-67: Ki-67 labeling index; MRI: Magnetic resonance imaging; SMA: a-Smooth muscle actin; US: Ultrasonography; VATS: Video-assisted thoracic surgery

\section{Acknowledgements}

We thank Prof. Akiyoshi Kakita for neuropathological examination.

\section{Authors' contributions}

$\mathrm{ES}$ and $\mathrm{HM}$ are the surgeons who operated on the patient. The manuscript was drafted by KM, ES, AN, HH, EG, and HM. KM supervised the preparation of this case report. All authors have read and approved the final manuscript.

\section{Funding}

Dr. Gabriel was supported by CTSA grant number KL2 TR002379 from the National Center for Advancing Translational Science (NCATS). The contents are solely the responsibility of the authors and do not necessarily represent the official views of the $\mathrm{NIH}$.

\section{Availability of data and materials}

Not applicable

Ethics approval and consent to participate

Not applicable

\section{Consent for publication}

Written informed consent was obtained from the patient's family for the publication of this case report and any accompanying images. A copy of the written consent is available for review by the Editor-in-Chief of this journal.

\section{Competing interests}

The authors declare that they have no competing interests.

\section{Author details}

${ }^{1}$ Department of Breast Surgery, Niigata City General Hospital, 463-7 Shumoku, Chuo-ku, Niigata 950-1197, Japan. ${ }^{2}$ Department of Pathology, Niigata City General Hospital, 463-7 Shumoku, Chuo-ku, Niigata 950-1197, Japan. ${ }^{3}$ Department of Pathology, Brain Research Institute, Niigata University, Niigata City 950-8585, Japan. ${ }^{4}$ Department of Surgery, Mayo Clinic, Jacksonville, FL 32224, USA. ${ }^{5}$ Makino Breast Clinic, Niigata 950-0861, Japan. 
Received: 24 January 2020 Accepted: 21 May 2020

Published online: 29 May 2020

\section{References}

1. Hamperl H. The myothelia (myoepithelial cells). Normal state; regressive changes; hyperplasia; tumors. Curr Top Pathol. Ergebnisse der Pathologie. 1970:53:161-220

2. Tavassoli FA. Myoepithelial lesions of the breast. Myoepitheliosis, adenomyoepithelioma, and myoepithelial carcinoma. Am J Surg Pathol. 1991;15:554-68.

3. Rasbridge SA, Millis RR. Adenomyoepithelioma of the breast with malignant features. Virchows Arch. 1998;432:123-30.

4. Kihara M, Yokomise H, Irie A, Kobayashi S, Kushida Y, Yamauchi A. Malignant adenomyoepithelioma of the breast with lung metastases: report of a case. Surg Today. 2001:31:899-903.

5. Bult P, Verwiel JM, Wobbes T, Kooy-Smits MM, Biert J, Holland R. Malignant adenomyoepithelioma of the breast with metastasis in the thyroid gland 12 years after excision of the primary tumor. Case report and review of the literature. Virchows Arch. 2000;436:158-66.

6. Takahashi II, Tashiro H, Wakasugi K, Onohara T, Nishizaki T, Ishikawa T, et al. Malignant adenomyoepithelioma of the breast: a case with distant metastases. Breast cancer. 1999:6:73-7.

7. Nadelman CM, Leslie KO, Fishbein MC. "Benign," metastasizing adenomyoepithelioma of the breast: a report of 2 cases. Arch Pathol Lab Med. 2006;130:1349-53.

8. Michal M, Baumruk L, Burger J, Manhalova M. Adenomyoepithelioma of the breast with undifferentiated carcinoma component. Histopathology. 1994; 24:274-6.

9. Petrozza V, Pasciuti G, Pacchiarotti A, Tomao F, Zoratto F, Rossi L. Breast adenomyoepithelioma: a case report with malignant proliferation of epithelial and myoepithelial elements. World J Surg Oncol. 2013;11:285.

10. Korolczuk A, Amarowicz M, Bak K, Korobowicz E, Koncewicz T. Adenomyoepithelioma of the breast with late pulmonary metastases - case report and review of the literature. J Cardiothorac Surg. 2016;11:121.

11. Hikino H, Kodama K, Yasui K, Ozaki N, Nagaoka S, Miura H. Intracystic adenomyoepithelioma of the breast--case report and review. Breast cancer. 2007:14:429-33.

12. Kim MJ, Kim CS, Ju MJ, Park YS. Malignant adenomyoepithelioma of the breast: a rare case report. Int J Surg Case Rep. 2019;59:111-4.

13. Nagao T, Sugano I, Ishida Y, Tajima Y, Matsuzaki O, Konno A, et al. Salivary gland malignant myoepithelioma: a clinicopathologic and immunohistochemical study of ten cases. Cancer. 1998:83:1292-9.

14. Ibiayi D, Alice S. Tumour heterogeneity and resistance to cancer therapies. Nat Rev Clin Oncol. 2018:15:81-94.

15. Lee S, Oh SY, Kim SH, Lee JH, Kim DC, Cho SH, et al. Malignant adenomyoepithelioma of the breast and responsiveness to eribulin. J Breast Cancer. 2015;18:400-3

\section{Publisher's Note}

Springer Nature remains neutral with regard to jurisdictional claims in published maps and institutional affiliations.

\section{Submit your manuscript to a SpringerOpen ${ }^{\circ}$ journal and benefit from:}

- Convenient online submission

- Rigorous peer review

- Open access: articles freely available online

High visibility within the field

- Retaining the copyright to your article

Submit your next manuscript at $\boldsymbol{\nabla}$ springeropen.com 